\title{
Preoperative Risk Score to Predict Occult Metastatic or Locally Advanced Disease in Patients with Resectable Perihilar Cholangiocarcinoma on Imaging
}

\author{
Jimme K Wiggers, MD, PhD ${ }^{1,2}$, Bas Groot Koerkamp, MD, $\mathrm{PhD}^{2,3}$, David van Klaveren, \\ PhD $^{4}$, Robert J Coelen, MD, PhD 1 , C Yung Nio, MD ${ }^{5}$, Peter J Allen, MD, FACS ${ }^{2}$, Marc G \\ Besselink, MD, PhD ${ }^{1}$, Olivier R Busch, MD, PhD ${ }^{1}$, Michael I D'Angelica, MD, FACS ${ }^{2}$, Ronald \\ P DeMatteo, MD, FACS ${ }^{2}$, T Peter Kingham, MD, FACS ${ }^{2}$, Thomas M van Gulik, MD, PhD ${ }^{1}$, and \\ William R Jarnagin, MD, FACS ${ }^{2}$
}

${ }^{1}$ Department of Surgery, Cancer Center Amsterdam, Academic Medical Center, the Netherlands ${ }^{2}$ Department of Surgery, Memorial Sloan Kettering Cancer Center, New York, NY ${ }^{3}$ Department of Surgery, Erasmus Medical Center, Rotterdam, the Netherlands ${ }^{4}$ Department of Epidemiology, Erasmus Medical Center, Rotterdam, the Netherlands ${ }^{5}$ Department of Radiology, Academic Medical Center, Amsterdam, the Netherlands

\section{Abstract}

Background: Many patients with resectable perihilar cholangiocarcinoma (PHC) on imaging are intra-operatively diagnosed with occult metastatic or locally advanced disease precluding a curative-intent resection. This study aimed to develop and validate a preoperative risk score.

Study Design: Patients with resectable PHC on imaging who underwent surgery in two highvolume centers (USA and Europe) between 2000 and 2015 were included. Multivariable logistic regression analysis was used to develop the risk score. Cross-validation was used to validate the score, alternating the two centers as 'training' and 'testing' dataset.

Results: Of 566 patients who underwent surgery 309 patients (55\%) underwent a resection, while in 257 patients (45\%) a curative-intent resection was precluded due to distant metastasis $(\mathrm{n}=151 ; 27 \%)$ or locally advanced disease $(\mathrm{n}=106 ; 19 \%)$. Preoperative predictors included bilirubin above $2 \mathrm{mg} / \mathrm{dL}$, bile duct involvement on imaging, portal vein involvement on imaging ( $\geq$ 180 degrees), hepatic artery involvement on imaging ( $\geq 180$ degrees), and suspicious lymph nodes on imaging. The new risk score (c-index 0.75 after cross-validation) provided significantly more accurate predictions than the Bismuth classification (c-index 0.62), Blumgart T-staging (c-index 0.67), and cTNM staging (c-index 0.68). The new risk score identified four risk groups for occult

Correspondence address: Jimme K. Wiggers, MD PhD, Department of Surgery, Cancer Center Amsterdamm, Academic Medical Center, Meibergdreef 9, 1105 AZ Amsterdam, the Netherlands, Jim.wiggers@ gmail.com, +31-6-43928312.

Publisher's Disclaimer: This is a PDF file of an unedited manuscript that has been accepted for publication. As a service to our customers we are providing this early version of the manuscript. The manuscript will undergo copyediting, typesetting, and review of the resulting proof before it is published in its final citable form. Please note that during the production process errors may be discovered which could affect the content, and all legal disclaimers that apply to the journal pertain.

Disclosure Information: Nothing to disclose.

Abstract presented at the meeting of the International Hepatobiliary Association, Sao Paulo, Brazil, April 2016. 
metastatic or locally advanced disease: low (14.7\%), intermediate (29.5\%), high (47.3\%), and very high risk $(81.3 \%)$. The preoperative score groups also predicted survival after surgery, irrespective of intra-operative findings $(P<0.001)$.

Conclusions: The validated risk score can predict occult distant metastatic or locally advanced PHC based on five preoperatively available factors. The score can be useful in preoperative shared decision-making and selection of patients in neo-adjuvant clinical trials.

\section{Precis}

Many patients with resectable perihilar cholangiocarcinoma on imaging are intraoperatively diagnosed with occult metastatic or locally advanced disease precluding a resection. This study of 566 patients developed and validated a preoperative risk score that can be used in shared decisionmaking and selection of patients in neoadjuvant trials.

\section{Keywords}

Perihilar cholangiocarcinoma; Klatskin; risk score; portal vein; hepatic artery; surgery; survival

\section{INTRODUCTION}

Curative-intent resection is the optimal treatment for perihilar cholangiocarcinoma (PHC), yielding a median overall survival of 20 to 40 months,(1) compared to 12 months with palliative chemotherapy alone.(2) Computed tomography (CT) and magnetic resonance cholangiopancreatography (MRCP) are both used to rule out metastatic disease and assess the local extent of the tumor prior to surgery.(3) However, about $40 \%$ of patients have distant metastatic or locally advanced disease diagnosed at surgical exploration, precluding a curative-intent resection. $(4,5)$ Preoperative prediction of the risk of occult metastatic or locally advanced disease could inform patients and physicians during shared decisionmaking.

Several preoperative staging systems have been developed for PHC focusing on the local extent of disease: the Bismuth classification, $(6,7)$ the Blumgart T-staging system, $(8,9)$ and the classification of the 'International Cholangiocarcinoma Group for the Staging of PHC'. (10) Also, the clinical TNM staging of the American Joint Committee on Cancer (AJCC) can be applied preoperatively to radiological parameters. $(11,12)$ These systems describe factors such as biliary extent of the tumor and vascular involvement on imaging. However, they were not based on statistical analysis of large patient series, and as such are not able to predict the risk of occult metastatic or locally advanced disease in individual patients.(13)

This study aimed to develop and validate a preoperative score that predicts the risk of an intra-operative diagnosis of distant metastatic or locally advanced disease in patients eligible for curative-intent surgery based on imaging. 


\section{METHODS}

\section{Patients}

Patients were included from prospective databases at Memorial Sloan Kettering Cancer Center (MSKCC, New York, USA) and the Academic Medical Center (AMC, Amsterdam, the Netherlands) between 2000 and 2015. PHC was defined as a biliary malignancy originating in the common hepatic duct, the hepatic duct confluence, or in the left or right hepatic duct. $(11,12)$ Preoperative imaging studies included CT, MRCP and/or PET-CT. Eligible patients had resectable PHC on imaging and underwent either a curative-intent resection or surgery (staging laparoscopy or exploratory laparotomy) without resection. Resectable PHC on imaging was defined as: no biopsy-proven distant metastasis and potentially resectable with radical (R0) margins. The estimated future liver remnant required a minimum of $30 \%$ (after portal vein embolization if necessary). The criteria to proceed with surgery and resection were similar in the two study centers during the study period. The study also included patients with presumed PHC, who were found to have benign disease after surgery. Inclusion of these patients reflects clinical practice, because $5-15 \%$ of patients will have unexpected benign disease at pathological examination of the resected specimen, which was unknown at the time of preoperative decision-making. $(14,15)$

\section{Surgery}

Preoperative biliary drainage was routinely used to relieve jaundice with endoscopic drainage, percutaneous drainage, or both. Patients often underwent staging laparoscopy first to evaluate the presence of peritoneal or liver metastasis, and if either was found, laparotomy was abandoned.

At laparotomy, suspected foci were sampled with frozen section histology. If feasible, resection consisted of hilar en-bloc resection of the extrahepatic bile ducts, combined with (extended) hemihepatectomy and caudate resection in most patients. Portal vein resection and reconstructions were used to obtain tumor-free resection margins if necessary and if technically feasible; hepatic artery resections requiring arterial reconstructions were rarely performed.

No resection was performed if distant metastatic or locally advanced disease was diagnosed during laparotomy. Distant metastatic disease was defined as Stage IV disease according to the $7^{\text {th }}$ edition of the AJCC staging system, including metastasis in N2 lymph nodes (i.e. aortocaval, superior mesenteric artery, and celiac artery lymph nodes) as stage IV disease. $(11,12)$ Locally advanced disease was defined as tumor that was not resectable with R0 or R1 margins due to extensive involvement of second-order bile ducts; due to insufficient $(<30 \%)$ or cirrhotic future liver remnant; due to non-reconstructable portal vein involvement; or due to involvement of the hepatic artery to the future liver remnant.

\section{Endpoint and predictor variables}

The endpoint of the analysis was an intra-operative diagnosis of distant metastatic or locally advanced PHC, either during staging laparoscopy or exploratory laparotomy. Definitions of preoperative predictor variables were based on the classification system from the 
International Cholangiocarcinoma Group for the staging of PHC.(10) All preoperative CT and MRCP studies were reassessed by a radiologist unaware of the clinical outcome. Vascular involvement (portal vein or hepatic artery) on imaging was defined as occlusion, narrowing, or circumferential tumor contact of at least 180 degrees. $(9,10)$ The extent of biliary involvement on imaging was classified according to the Bismuth classification. $(6,7)$ Tumor size on imaging was defined as largest diameter in longitudinal or radial measurement (whichever was larger), and classified as not detectable or smaller than $1 \mathrm{~cm}$, between 1 and $3 \mathrm{~cm}$, or at least $3 \mathrm{~cm}$.(10) Lymph nodes were classified as suspicious if they were larger than $10 \mathrm{~mm}$ in short-axis diameter or if they had central necrosis on imaging. (16) Lobar atrophy was defined as a small, often hypoperfused lobe with crowding of dilated intrahepatic bile ducts.(8) Demographics and bilirubin levels were collected from the medical charts. A peak total bilirubin level of at least $2 \mathrm{mg} / \mathrm{dL}$ (equivalent to $34 \mu \mathrm{mol} / \mathrm{L}$ ) prior to biliary drainage was used as a cut-off, reflecting biliary obstruction by the tumor.

\section{Data analysis}

Data are presented as mean \pm standard deviation (SD) or proportion and percentage, as appropriate. Missing data were imputed with multiple imputation (5 imputation cohorts). Variables with missing data included suspicious lymph nodes $(\mathrm{n}=22 ; 3.9 \%)$, portal vein involvement on imaging $(\mathrm{n}=21 ; 3.7 \%)$, hepatic artery involvement on imaging $(\mathrm{n}=36 ; 6.4 \%)$, lobar atrophy $(\mathrm{n}=18 ; 3.2 \%)$, and tumor size on imaging $(\mathrm{n}=32 ; 5.7 \%)$. A regression model was used with all study variables, and the data was pooled using Rubin's rule. Statistical analyses were performed with R software version 2.13.1 (R Foundation for Statistical Computing, Vienna, Austria) and SPSS version 21.0 (SPSS, Inc., Chicago, Illinois).

\section{Model development and validation}

Predictor variables from all patients were entered in a multivariable logistic regression model. Categories within the study variables were subsequently combined if they had comparable odds ratios. Backward selection was used, and variables with a p-value above 0.05 were removed from the final model. The discriminative ability of the model was assessed with area under the curve (AUC) analysis and reported as c-statistics, which was corrected for optimism using bootstrap resampling (100 models). The model was validated with cross-validation, alternating the two centers as 'training' and 'testing' dataset.(17, 18)

\section{Preoperative risk score}

The model is presented as a risk score. Scores were calculated by dividing the products of regression coefficients and predictor values by the smallest product. A constant was subtracted or added to rescale the scores in positive integers. The sum scores were then related to the risk of occult distant metastatic or locally advanced disease. Comparisons with the Bismuth, Blumgart, and cTNM staging systems were performed with area under the curve analysis and reported as c-statistics. Statistical significance between c-statistics was tested with the method of Hanley \& McNeill.(19) Overall survival (OS) of four risk groups was assessed with Kaplan-Meier analysis and compared with the log-rank test. OS was determined between the date of surgery and the date of death or last follow-up. 


\section{RESULTS}

\section{Patients}

A total of 566 patients were included in the study. Baseline characteristics of the study populations are shown in Table 1. The populations from MSKCC $(n=277)$ and AMC $(n=289)$ differed in age, peak bilirubin level, suspicious lymph nodes on imaging, extent of biliary tumor involvement on imaging (Bismuth classification), tumor size on imaging, and lobar atrophy on imaging. The populations also differed in the use of preoperative biliary drainage, MRCP, and staging laparoscopy.

\section{Surgical procedures and diagnosis}

Figure 1 shows the postoperative outcomes. Staging laparoscopy was used in 399 patients (70.5\%), revealing histopathological evidence of distant metastasis in 56 patients (14.0\%) and locally advanced disease in 9 patients (2.3\%). Of 501 patients who underwent exploratory laparotomy (88.5\%), 95 patients were diagnosed with distant metastasis (19.0\%) and 97 patients with locally advanced disease (19.4\%). In total, a curative-intent resection was precluded in 257 patients (45.4\%). Distant metastasis precluding a resection concerned $\mathrm{N} 2$ lymph nodes metastasis in 61 patients (10.8\%), intrahepatic in 27 patients (4.8\%), peritoneum in 44 patients (7.8\%), and multiple sites in 19 patients (3.4\%). Locally advanced disease precluding a curative-intent resection concerned vascular involvement in 66 patients $(11.7 \%)$, biliary involvement in 33 patients (5.8\%), and both in 7 patients (1.2\%).

A curative-intent resection was performed in 309 patients (54.6\%). Extrahepatic bile duct resection alone was performed in 53 patients (17.1\%). Extrahepatic bile duct resection was combined with a left (extended) hepatectomy in 120 patients (38.8\%), a right (extended) hepatectomy in 124 patients (40.1\%), a central hepatectomy in 3 patients (0.9\%), a segment $4 / 5$ wedge resection in 9 patients (2.9\%). Caudate resection was used in 159 of 247 patients with a major hepatectomy (64.4\%), and more often between 2010-2015 (70.8\%) compared to 2000-2009 (62.4\%). A combined pancreatoduodenectomy was performed in 9 patients (2.9\%), and portal vein reconstruction was used in 49 patients (15.9\%).

After resection, the diagnosis of PHC was confirmed in 268 of 309 patients (86.7\%). The resection specimen revealed cholangiocarcinoma in the distal bile ducts in 5 patients (1.6\%), gallbladder carcinoma in 5 patients (1.6\%), other malignancy in 4 patients $(1.3 \%)$, and a benign stricture in 27 patients (8.7\%). Among 282 patients submitted to curative intent resection for malignancy, definitive resection margins were tumor-negative (R0) in 220 (78.0\%).

\section{Multivariable analysis}

The multivariable analysis of all predictor variables in the whole cohort $(n=566)$ is shown in eTable 1. Preoperative bilirubin above $2 \mathrm{mg} / \mathrm{dL}$, extent of bile duct involvement on imaging, suspected portal vein involvement on imaging, suspected hepatic artery involvement on imaging, and suspicious lymph nodes on imaging were independent predictors and were retained in the final model, which is shown in Table 2. Lobar atrophy and tumor size on imaging were not independent poor prognostic factors (eTable 1). 


\section{Model validation}

The optimism corrected c-index of the final model was 0.75 (95\% confidence interval (CI) 0.71-0.79). Cross-validation with re-estimation of the model parameters in the MSKCC cohort and validation in the AMC cohort yielded a c-index of 0.75 (95\% CI 0.70-0.81). Cross-validation with re-estimation in the AMC cohort and validation in the MSKCC cohort resulted in a c-index of 0.76 (95\% CI 0.70-0.82). Calibrations of predicted risks after crossvalidation are shown in eFigure 1.

\section{Preoperative score}

The preoperative score based on the final model is presented in Table 2. The sum of the score ranges between 0 and 16 points; the predicted risks according to the score are shown in Figure 2. Based on ranking in quartiles, four risk groups were identified: low ( $0-3$ points; risk $14.7 \%$ ), intermediate (4 points; risk $29.5 \%$ ), high (5-6 points; risk $47.3 \%$ ), and very high ( $\geq 7$ points; risk $81.3 \%$ ). The c-index of the preoperative score groups was 0.75 (95\% CI 0.71-0.79), which was significantly higher compared to other preoperative staging systems, including the Bismuth classification (c-index 0.62, 95\% CI 0.57-0.67, $P<0.001$ ), the Blumgart T-staging system (c-index 0.67, 95\% CI 0.63-0.71, $P=0.01$ ), and the cTNM staging system (c-index $0.68,95 \%$ CI $0.63-0.72, P=0.02$ ).

\section{Positive predictive values}

Table 3 shows the positive predictive values of the individual predictor variables and the several staging systems, including the new preoperative risk score classes The predictor variables included in the new preoperative score, which are based on locoregional tumor factors, were also predictive for occult distant metastasis. No risk factor had a 100\% positive predictive value to predict occult locally advanced or metastatic disease (i.e. there was no risk factor with $0 \%$ resections).

\section{Postoperative mortality and survival}

After exploratory laparotomy, postoperative mortality within 90 days occurred in 49 of 501 patients $(9.7 \%)$, consisting of 32 of 309 patients (10.4\%) who underwent a resection and 17 of 192 patients $(8.9 \%)$ who did not undergo a resection. The median OS of all 566 patients was 19.7 months. At last follow-up, 405 patients (71.6\%) had died; median follow-up for patients alive at last follow-up was 31 months. The preoperative risk groups had different OS; median OS was 53.2 months in the low risk group, 22.1 months in the intermediate risk group, 19.2 months in the high risk group, and 11.5 months in the very high risk group. $(P<0.001$, Figure 3$)$.

\section{DISCUSSION}

This study presents a validated preoperative risk score for occult distant metastatic or locally advanced disease in patients with suspected PHC. The score considers bilirubin level, extent of biliary involvement on imaging, vascular involvement on imaging, and suspicious lymph nodes on imaging. Patients were classified in four risk groups: low (14.7\%), intermediate (29.5\%), high (47.3\%), and very high risk (81.3\%). The score had a good discriminative ability that was significantly superior to the Bismuth, Blumgart and cTNM staging systems. 
It also predicted for overall survival after surgery, irrespective of intra-operative findings and resections.

Previous preoperative staging systems for PHC were not based on statistical analysis of patient data. The Bismuth classification stems from 1975 and is traditionally used to describe the extent of tumor involvement of the bile ducts, $(6,7)$ but it was not designed to predict distant metastatic or locally advanced disease. The Blumgart T-staging system was designed in 1998 and modified in 2001, and takes into account the extent of biliary involvement, portal vein involvement, and lobar atrophy. $(8,9)$ However, the present study showed that unilateral portal vein involvement and lobar atrophy are not independent predictors of occult metastatic or locally advanced disease. The recent Mayo staging system stratifies all patients that present with PHC into prognostic classes regardless of subsequent treatment.(20) It did not aim to predict occult metastatic or locally advanced disease and performed insufficient in the prediction of survival in patients who underwent surgery (cindex 0.57 in the derivation study and 0.56 in an external validation study). $(20,21)$ The conventional TNM staging system is often used to stage patients after postoperative pathology.(11) However, when it was applied to preoperative radiological parameters in this study it performed inferior to the presented new preoperative score. Staging included CT in most patients (96\%), and MRCP in more than half of the patients (54\%). Both modalities have individual advantages as long as the images are made prior to stent insertion, although some surgeons prefer preoperative MRCP to more accurately assess longitudinal tumor growth along the bile ducts. PET-CT scans were made in approximately $20 \%$ of the patients. There is no clear data to show a benefit of preoperative PET-CT, but it may help to further analyze potential metastasis pre-operatively. $(22,23)$

None of the preoperative risk factors in this study had a $100 \%$ positive predictive value to predict occult locally advanced or metastatic PHC, indicating that neither of these risk factors predicted a $0 \%$ chance of a resection. The multidisciplinary team meeting to pursue a resection should therefore be multifactorial, taking into account a patients' age, physical status, and tumor characteristics on imaging. The presented preoperative risk score may help to guide patient selection for surgery. Patients with low (14.7\%) or intermediate $(29.5 \%)$ risk of occult locally advanced or metastatic disease should undergo exploration. Patients with a high $(47.3 \%)$ or very high risk $(81.3 \%)$ should undergo staging laparoscopy first, and proceed to exploratory laparotomy only if feasible.(24) Moreover, fragile patients with high age and/or poor physical status and a high or very high risk of occult locally advanced or metastatic PHC based on the risk score should start with optimal palliative treatment instead of surgery.

There is currently limited evidence available to support neoadjuvent therapy for PHC.(25) A systematic review of retrospective studies suggested that neoadjuvant chemotherapy for advanced PHC is safe,(26) and case series have described successful downstaging with chemoradiotherapy and subsequent $\mathrm{R} 0$ resection of locally advanced cholangiocarcinoma. (27-29) Prospective studies to assess neoadjuvant treatment for PHC are needed; such studies could use the preoperative score to select patients with a high or very high risk of occult metastatic or locally advanced disease. These patients might benefit from 
(radio)chemotherapy as a first treatment option, and subsequent resection if feasible after response evaluation.

This study has several strengths. It is based on 566 consecutive patients from two highvolume centers in Europe and the USA. The prognostic factors were defined according to the classification system of the 'International Cholangiocarcinoma Group for the Staging of PHC',(10) which was designed to standardize the reporting of PHC. Radiologic images of all patients in the study were re-assessed by blinded radiologists to adhere in particular to the definition of vascular involvement (occlusion, narrowing, or contact of at least 180 degrees). The strictly defined parameters in the study should minimize interobserver variability, and thus increase reproducibility of the risk score. The derivation and validation of the risk score was performed according to the current state of the art methodology. In particular, the data of the two centers was purposefully not split in a separate derivation and validation dataset, since that method results in less accurate and less precise models. Instead, a full cohort multivariable analysis was performed, and cross-validation was used to validate the model. $(17,18)$ Despite differences in baseline characteristics between patients from the two centers, the risk score performed equally well in both centers during validation.

Limitations of this study include its retrospective study design and long study period. The quality of the preoperative imaging studies varied during the study period, and indications for preoperative biliary drainage, portal vein embolization, and surgery are naturally shifting. Nonetheless, all patients were preoperatively analyzed with CT and/or MRCP, and these images were re-reviewed in a blinded fashion for this study, ensuring homogeneity regarding the risk score variables.

\section{CONCLUSIONS}

This study shows that radiological parameters correlate to the risk of occult locally advanced or metastatic PHC diagnosed at surgery. The presented preoperative score can help patients and their physicians during shared decision-making when surgery for PHC is considered, and may be useful in the design of neoadjuvant clinical trials.

\section{Acknowledgments}

Support: Supported in part by NIH/NCI P30 CA008748 (Cancer Center Support Grant). Dr Wiggers was funded by the Academic Medical Center Foundation, and Dr Groot Koerkamp was funded by the Dutch Cancer Society (DCS), grant number UVA 2011-4973.

\section{ABBREVIATIONS}

$\begin{array}{ll}\text { PHC } & \text { perihilar cholangiocarcinoma } \\ \text { CT } & \text { computed tomography } \\ \text { PET-CT } & \text { Positron emission tomography-computed tomography } \\ \text { MRCP } & \text { magnetic resonance cholangiopancreatography } \\ \text { TNM staging } & \text { tumor-node-metastasis staging }\end{array}$


AJCC

MSKCC

AMC

AUC

OS
American Joint Committee on Cancer

Memorial Sloan Kettering Cancer Center

Academic Medical Center

area under the curve

overall survival

\section{REFERENCES}

1. Popescu I , Dumitrascu T . Curative-intent surgery for hilar cholangiocarcinoma: prognostic factors for clinical decision making. Langenbecks Arch Surg 2014;399:693-705.24841192

2. Valle J, Wasan H, Palmer DH, et al. Cisplatin plus gemcitabine versus gemcitabine for biliary tract cancer. N Engl J Med 2010;362:1273-1281.20375404

3. Park HS , Lee JM , Choi J-Y , et al. Preoperative evaluation of bile duct cancer: MRI combined with MR cholangiopancreatography versus MDCT with direct cholangiography. Am J Roentgenol 2008;190:396-405.18212225

4. Matsuo K, Rocha FG , Ito K, et al. The Blumgart preoperative staging system for hilar cholangiocarcinoma: analysis of resectability and outcomes in 380 patients. J Am Coll Surg 2012;215:343-355.22749003

5. Ruys AT , Busch OR, Rauws EA, et al. Prognostic impact of preoperative imaging parameters on resectability of hilar cholangiocarcinoma. HPB Surg 2013;2013:657309.23861556

6. Bismuth H , Corlette MB . Intrahepatic cholangioenteric anastomosis in carcinoma of the hilus of the liver. Surg Gynecol Obstet 1975;140:170-178.1079096

7. Bismuth H, Nakache R, Diamond T . Management strategies in resection for hilar cholangiocarcinoma. Ann Surg 1992;215:31-38.1309988

8. Burke EC , Jarnagin WR, Hochwald SN , et al. Hilar Cholangiocarcinoma: patterns of spread, the importance of hepatic resection for curative operation, and a presurgical clinical staging system. Ann Surg 1998;228:385-394.9742921

9. Jarnagin WR , Fong Y, DeMatteo RP, et al. Staging, resectability, and outcome in 225 patients with hilar cholangiocarcinoma. Ann Surg 2001;234:507-517; discussion 517-519.11573044

10. Deoliveira ML, Schulick RD, Nimura Y, et al. New staging system and a registry for perihilar cholangiocarcinoma. Hepatology 2011;53:1363-1371.21480336

11. Edge SB . AJCC cancer staging manual New York: Springer; 2010.

12. Compton CC . AJCC cancer staging atlas a companion to the seventh editions of the AJCC cancer staging manual and handbook New York, NY Available at: file://Users/Jimme/Documents/ Cholangiocarcinoma.sente6lib/Contents/Attachments/Compton,\%20CC/2012/AJCC\%20cancer $\% 20$ staging\%20atlas\%20a\%20co.pdf.

13. Ismael HN , Loyer E, Kaur H, et al. Evaluating the clinical applicability of the European staging system for perihilar cholangiocarcinoma. J Gastrointest Surg 2016;20:741-747.26801328

14. Corvera CU , Blumgart LH , Darvishian F, et al. Clinical and pathologic features of proximal biliary strictures masquerading as hilar cholangiocarcinoma. J Am Coll Surg 2005;201:862869.16310689

15. Erdogan D , Kloek JJ , ten Kate FJW , et al. Immunoglobulin G4-related sclerosing cholangitis in patients resected for presumed malignant bile duct strictures. Br J Surg 2008;95:727734.18418862

16. Lee HY, Kim SH , Lee JM , et al. Preoperative assessment of resectability of hepatic hilar cholangiocarcinoma: combined CT and cholangiography with revised criteria. Radiology 2006;239:113-121.16467211

17. Austin PC, van Klaveren D, Vergouwe Y, et al. Geographic and temporal validity of prediction models: different approaches were useful to examine model performance. J Clin Epidemiol 2016;79:76-85.27262237 
18. Cho HJ , Kim B , Lee J-D , et al. Development of risk prediction model for hepatocellular carcinoma progression of indeterminate nodules in hepatitis B virus-related cirrhotic liver. Am J Gastroenterol 2017;112:460-470.27779194

19. Hanley JA , McNeil BJ . The meaning and use of the area under a receiver operating characteristic (ROC) curve. Radiology 1982;143:29-36.7063747

20. Chaiteerakij R, Harmsen WS , Marrero CR, et al. A new clinically based staging system for perihilar cholangiocarcinoma. Am J Gastroenterol 2014;109:1881-1890.25384902

21. Coelen RJS , Gaspersz MP , Labeur TA, et al. Validation of the Mayo Clinic staging system in determining prognoses of patients with perihilar cholangiocarcinoma. Clin Gastroenterol Hepatol 2017519.

22. Kim JY , Kim M-H , Lee TY, et al. Clinical role of 18F-FDG PET-CT in suspected and potentially operable cholangiocarcinoma: a prospective study compared with conventional imaging. Am J Gastroenterol 2008;103:1145-1151.18177454

23. Petrowsky H, Wildbrett P, Husarik DB , et al. Impact of integrated positron emission tomography and computed tomography on staging and management of gallbladder cancer and cholangiocarcinoma. J Hepatology 2006;45:43-50.

24. Coelen RJS , Ruys AT, Wiggers JK, et al. Development of a risk score to predict detection of metastasized or locally advanced perihilar cholangiocarcinoma at staging laparoscopy. Ann Surg Oncol 2016;23:904-910.27586005

25. Weiss MJ , Cosgrove D , Herman JM , et al. Multimodal treatment strategies for advanced hilar cholangiocarcinoma. Langenbecks Arch Surg 2014;399:679-692.24962146

26. Grendar J , Grendarova P, Sinha R, Dixon E . Neoadjuvant therapy for downstaging of locally advanced hilar cholangiocarcinoma: a systematic review. HPB (Oxford) 2014;16:297303.23981000

27. McMasters KM , Tuttle TM , Leach SD, et al. Neoadjuvant chemoradiation for extrahepatic cholangiocarcinoma. Am J Surg 1997;174:605-608; discussion 608-609.9409582

28. Jung JH , Lee HJ , Lee HS , et al. Benefit of neoadjuvant concurrent chemoradiotherapy for locally advanced perihilar cholangiocarcinoma. World J Gastroenterol 2017 14;23:3301-3308.28566890

29. Le Roy B , Gelli M , Pittau G, et al. Neoadjuvant chemotherapy for initially unresectable intrahepatic cholangiocarcinoma. Br J Surg 2017831.

J Am Coll Surg. Author manuscript; available in PMC 2019 August 01. 
Patients with presumed PHC eligible for resection based on imaging

$\mathrm{n}=566$

$n=277$ from $M S K C C$ and $n=289$ from $A M C$

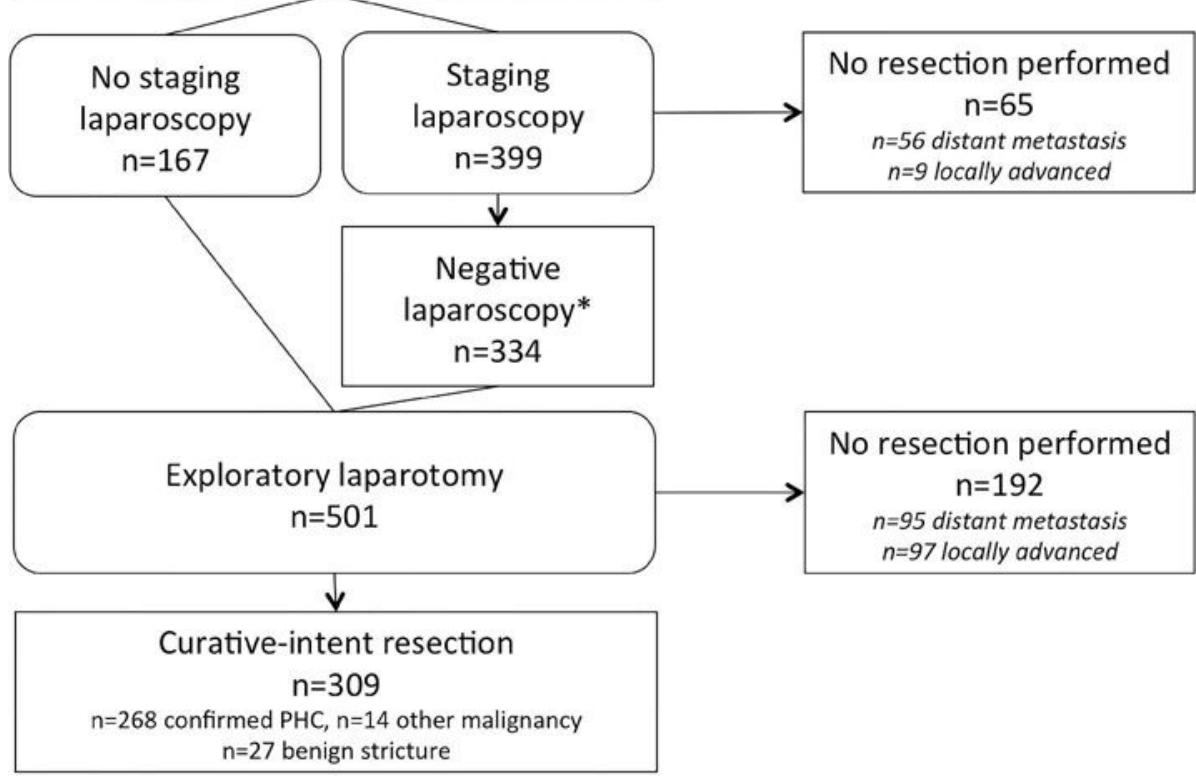

Figure 1.

Flowchart of patients in the study. *No evidence of distant metastasis or locally advanced disease during staging laparoscopy. AMC, Academic Medical Center Amsterdam; MSKCC, Memorial Sloan Kettering Cancer Center; PHC, perihilar cholangiocarcinoma. 


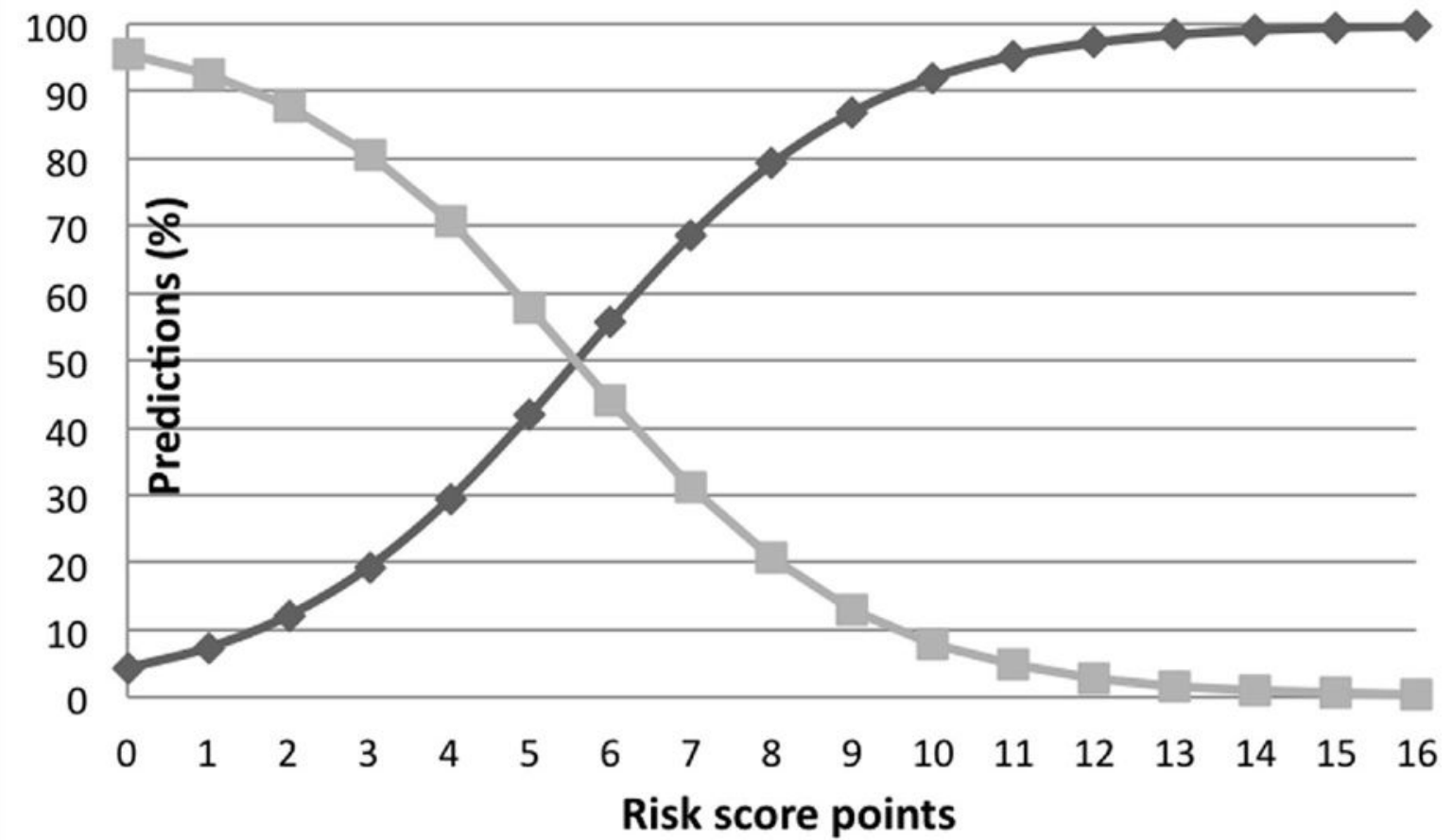

$\sim$ Risk of distant metastases or locally advanced disease - Chance of a curative-intent resection

Figure 2.

Predictions according to the preoperative score for perihilar cholangiocarcinoma. Presented are the risks of occult metastatic or locally advanced disease for each point score, as well as the inverted risks, which reflect the chance that a curative-intent resection can be performed. 


\section{Risk groups}

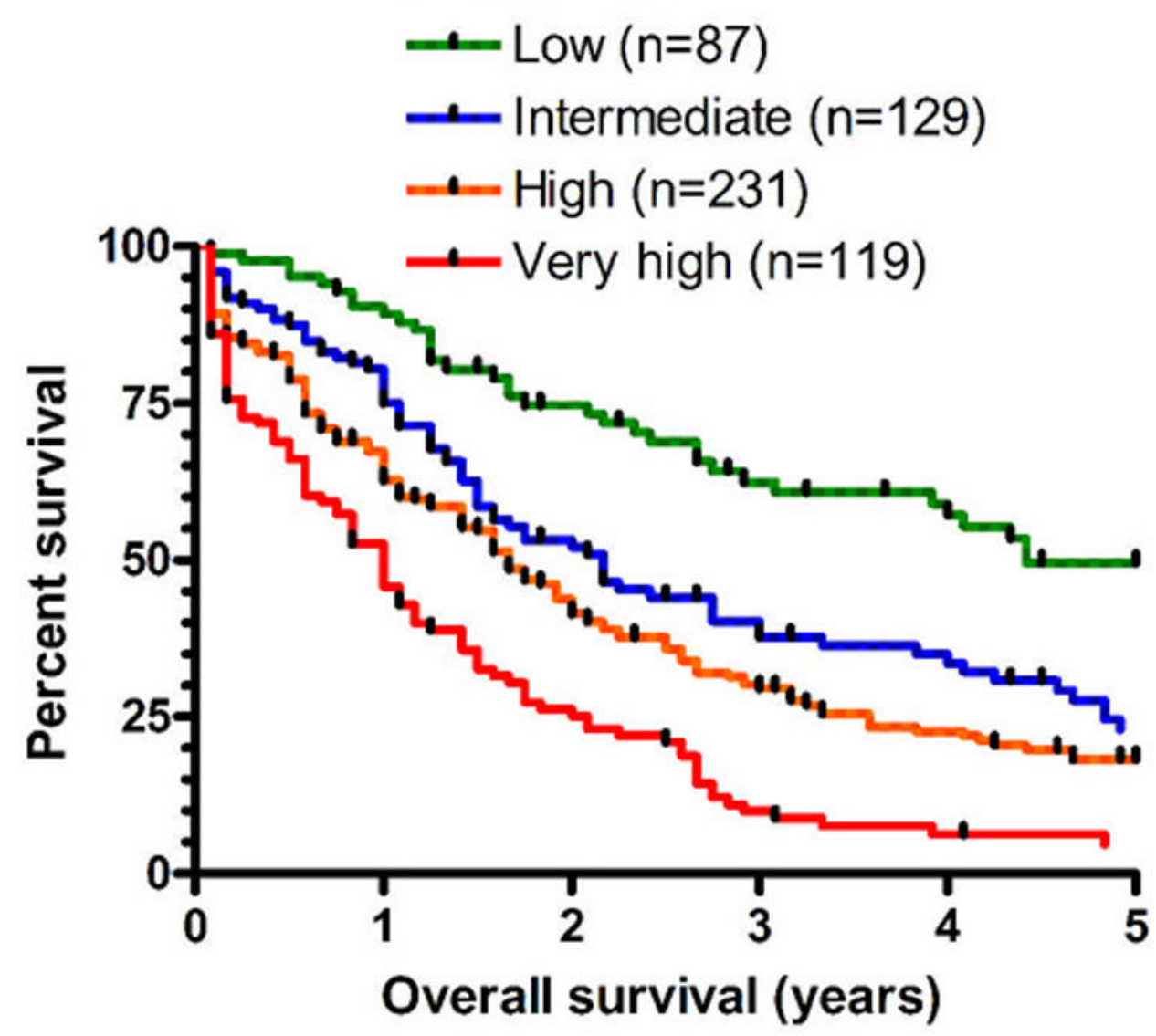

Patients at risk (n)

$\begin{array}{rrrrrr}87 & 75 & 52 & 39 & 31 & 20 \\ 129 & 93 & 49 & 35 & 24 & 16 \\ 231 & 137 & 77 & 50 & 31 & 21 \\ 119 & 60 & 26 & 10 & 5 & 4\end{array}$

Figure 3.

Postoperative survival stratified according to the preoperative risk score groups. 


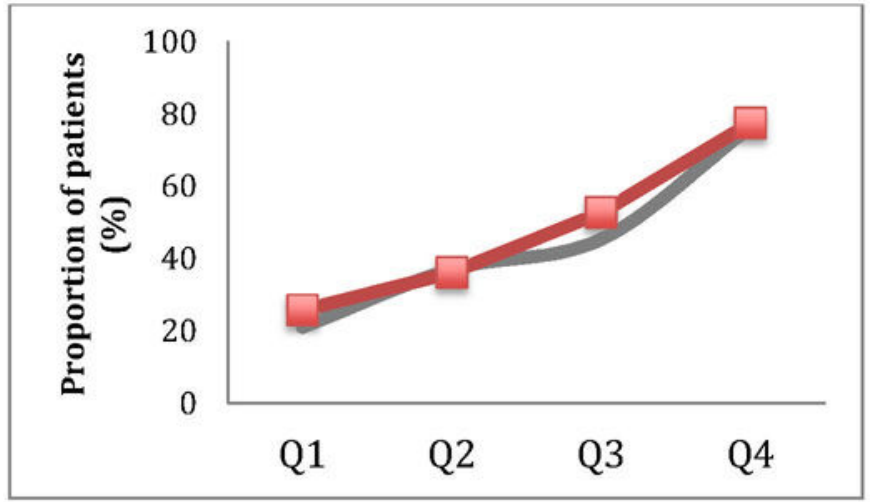

A

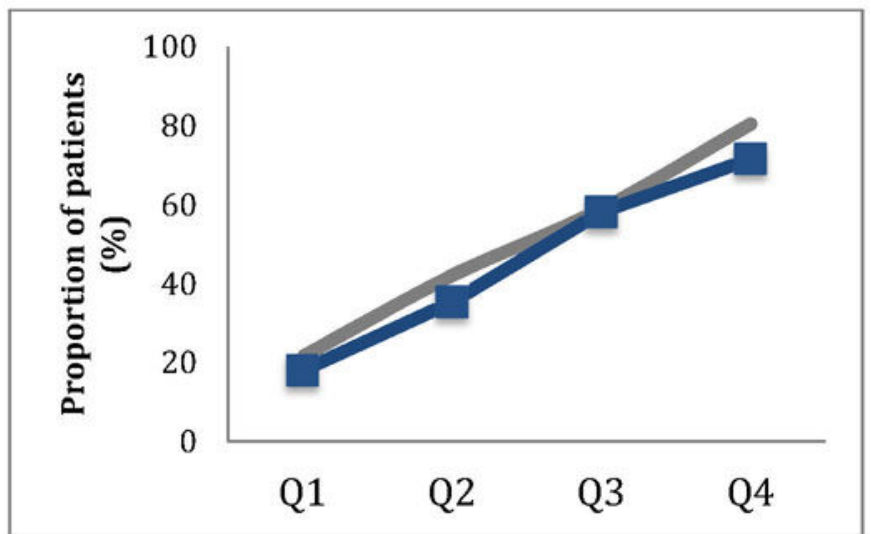

B

eFigure 1.

Calibration of the final model after cross-validation. Grey lines represent predicted outcomes; color lines with marks represent observed outcomes. (A) Predicted outcomes based on the MSKCC cohort and calibration of observed outcomes in quartiles of the AMC cohort. (B) Predicted outcomes based on the AMC cohort and calibration of observed outcomes in quartiles of the MSKCC cohort. AMC, Academic Medical Center Amsterdam; MSKCC, Memorial Sloan Kettering Cancer Center; Q, quartile of patients. 
Table 1.

Baseline Characteristics of the Study Groups

\begin{tabular}{|c|c|c|c|c|}
\hline Characteristic & $\begin{array}{l}\text { All patients } \\
(\mathrm{n}=566)\end{array}$ & $\begin{array}{l}\text { MSKCC cohort } \\
(\mathbf{n}=277)\end{array}$ & $\begin{array}{l}\text { AMC cohort } \\
(n=289)\end{array}$ & $\begin{array}{l}\text { p } \\
\text { Value }\end{array}$ \\
\hline Age, mean (SD) & $65(11)$ & $66(12)$ & $63(11)$ & 0.01 \\
\hline Male, n (\%) & $351(62.0)$ & $165(59.6)$ & $186(64.4)$ & 0.26 \\
\hline $\begin{array}{l}\text { Peak bilirubin }>2 \mathrm{mg} / \mathrm{dL} \text {, before } \\
\text { biliary drainage, } \mathrm{n}(\%)\end{array}$ & $459(81.1)$ & $215(77.6)$ & $244(84.4)$ & 0.04 \\
\hline $\begin{array}{l}\text { Preoperative biliary drainage, } \mathrm{n} \\
(\%)\end{array}$ & $487(86.0)$ & $224(80.9)$ & $269(91.0)$ & $<0.001$ \\
\hline \multicolumn{5}{|l|}{ Preoperative staging, n (\%) } \\
\hline $\mathrm{CT}$ & $545(96.3)$ & $266(96.0)$ & $279(96.5)$ & 0.83 \\
\hline MRCP & $304(53.7)$ & $203(73.7)$ & $101(34.9)$ & $<0.001$ \\
\hline PET & $120(21.2)$ & $59(21.3)$ & $61(21.1)$ & 0.99 \\
\hline Staging laparoscopy & $399(70.5)$ & $171(61.7)$ & $228(78.9)$ & $<0.001$ \\
\hline $\begin{array}{l}\text { Bismuth classification on imaging, } \\
\mathrm{n}(\%)^{*}\end{array}$ & & & & 0.01 \\
\hline Left or right duct only & $35(6.2)$ & $14(5.1)$ & $21(7.3)$ & \\
\hline B1 & $69(12.2)$ & $42(15.2)$ & $27(9.3)$ & \\
\hline B2 & $55(9.7)$ & $24(8.7)$ & $31(10.7)$ & \\
\hline $\mathrm{B} 3 \mathrm{~A}$ - right & $172(30.4)$ & $71(25.6)$ & $101(34.9)$ & \\
\hline B3B - left & $109(19.3)$ & $52(18.8)$ & $57(19.7)$ & \\
\hline B4 & $126(22.3)$ & $74(26.7)$ & $52(18.0)$ & \\
\hline $\begin{array}{l}\mathrm{PV} \text { involvement on imaging, } \mathrm{n} \\
(\%)^{\dagger t^{t}}\end{array}$ & & & & 0.09 \\
\hline None & $268(47.3)$ & $119(43.0)$ & $149(51.6)$ & \\
\hline Unilateral & $215(38.0)$ & $117(42.2)$ & $98(33.9)$ & \\
\hline Bilateral or main & $62(11.0)$ & $30(10.8)$ & $32(11.1)$ & \\
\hline $\begin{array}{l}\text { HA involvement on imaging, } \mathrm{n} \\
(\%)^{\dagger \psi^{t}}\end{array}$ & & & & 0.36 \\
\hline None & $330(58.3)$ & $163(58.8)$ & $167(57.8)$ & \\
\hline Unilateral & 169 (29.9) & 74 (26.7) & $95(32.9)$ & \\
\hline Bilateral or main & $31(5.5)$ & $17(6.1)$ & $14(4.8)$ & \\
\hline Suspicious LN on imaging, $\mathrm{n}(\%)^{t^{t}}$ & & & & $<0.001$ \\
\hline None & $290(51.2)$ & $110(39.7)$ & $180(62.3)$ & \\
\hline N1 & $199(35.2)$ & $120(43.3)$ & $79(27.3)$ & \\
\hline $\mathrm{N} 2$ & $55(9.7)$ & $33(11.9)$ & $22(7.6)$ & \\
\hline Tumor size on imaging, $\mathrm{n}(\%)^{*}$ & & & & 0.003 \\
\hline$<1 \mathrm{~cm}$ & $61(10.8)$ & $42(15.2)$ & $19(6.6)$ & \\
\hline $1-3 \mathrm{~cm}$ & $292(51.6)$ & $139(50.2)$ & $153(52.9)$ & \\
\hline$>3 \mathrm{~cm}$ & $181(32.0)$ & $79(28.5)$ & $102(35.3)$ & \\
\hline
\end{tabular}

J Am Coll Surg. Author manuscript; available in PMC 2019 August 01. 


\begin{tabular}{|l|l|l|l|l|}
\hline Characteristic & $\begin{array}{l}\text { All patients } \\
(\mathbf{n = 5 6 6 )}\end{array}$ & $\begin{array}{l}\text { MSKCC cohort } \\
(\mathbf{n = 2 7 7 )}\end{array}$ & $\begin{array}{l}\text { AMC cohort } \\
(\mathbf{n = 2 8 9})\end{array}$ & $\begin{array}{l}\mathbf{p} \\
\text { Value }\end{array}$ \\
\hline Lobar atrophy on imaging, n (\%) & $189(33.4)$ & $109(39.4)$ & $80(27.7)$ & 0.002 \\
\hline Portal vein embolization, $\mathrm{n}(\%)$ & $12(2.1)$ & $2(0.7)$ & $10(3.5)$ & 0.08 \\
\hline
\end{tabular}

Bismuth classification describes the extent of bile duct involvement.

${ }^{\dagger}$ Vascular involvement (portal vein or hepatic artery) on imaging was defined as occlusion, narrowing, or circumferential tumor contact of at least 180 degrees.

${ }^{*}$ Numbers do not sum to group totals due to missing data.

AMC, Academic Medical Center Amsterdam; HA, hepatic artery; LN, lymph nodes; MRCP, magnetic resonance cholangiopancreatography; MSKCC, Memorial Sloan Kettering Cancer Center; PET, positron emission tomography; PV, portal vein. 
Table 2.

Final Multivariable Model and Preoperative Score for Perihilar Cholangiocarcinoma

\begin{tabular}{|c|c|c|c|c|}
\hline \multirow[t]{2}{*}{ Variable } & \multicolumn{3}{|c|}{ Final model } & \multirow{2}{*}{$\begin{array}{l}\text { Preoperative } \\
\text { score points }\end{array}$} \\
\hline & $\begin{array}{l}\text { Odds } \\
\text { ratio }\end{array}$ & $95 \%$ CI & $\underset{\text { Value }}{\mathbf{p}}$ & \\
\hline \multicolumn{5}{|l|}{ Bilirubin $>2 \mathrm{mg} / \mathrm{dL}$} \\
\hline No & Reference & - & - & 0 \\
\hline Yes & 1.89 & $1.09-3.26$ & 0.02 & 1 \\
\hline \multicolumn{5}{|l|}{ Bismuth classification on imaging ${ }^{*}$} \\
\hline Left or right hepatic duct only & 0.39 & $0.10-1.47$ & 0.17 & 0 \\
\hline B1 & Reference & - & - & 2 \\
\hline $\mathrm{B} 2$ or $3 \mathrm{~A}$ or $3 \mathrm{~B}$ & 2.04 & $1.05-3.93$ & 0.04 & 3 \\
\hline B4 & 2.74 & $1.30-5.76$ & 0.01 & 4 \\
\hline \multicolumn{5}{|l|}{$\mathrm{PV}$ involvement on imaging ${ }^{\dagger}$} \\
\hline None & Reference & - & - & 0 \\
\hline Unilateral & 1.20 & $0.77-1.88$ & 0.42 & 0 \\
\hline Bilateral or main stem & 4.96 & $2.33-10.57$ & $<0.001$ & 3 \\
\hline \multicolumn{5}{|l|}{ HA involvement on imaging ${ }^{\dagger}$} \\
\hline None & Reference & - & - & 0 \\
\hline Unilateral & 1.81 & $1.16-2.83$ & 0.01 & 1 \\
\hline Bilateral or main stem & 16.96 & $2.97-85.89$ & 0.001 & 6 \\
\hline \multicolumn{5}{|l|}{ Suspicious LN on imaging } \\
\hline None & Reference & - & - & 0 \\
\hline N1 & 1.87 & $1.24-2.81$ & 0.003 & 1 \\
\hline $\mathrm{N} 2$ & 2.78 & $1.44-5.38$ & 0.002 & 2 \\
\hline
\end{tabular}

Higher point scores represent higher risks of occult distant metastatic or locally advanced disease.

The risks corresponding to total point scores are shown in Figure 3.

Bismuth classification describes the extent of bile duct involvement.

${ }^{\dagger}$ Vascular involvement (portal vein or hepatic artery) on imaging was defined as occlusion, narrowing, or circumferential tumor contact of at least 180 degrees.

HA, hepatic artery; LN, lymph nodes; PV, portal vein. 


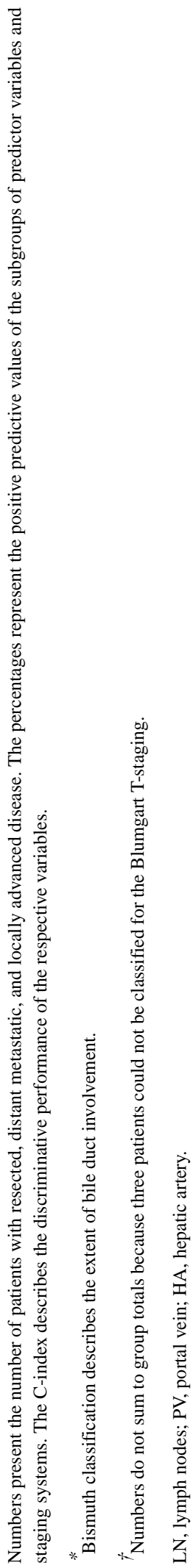

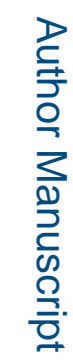
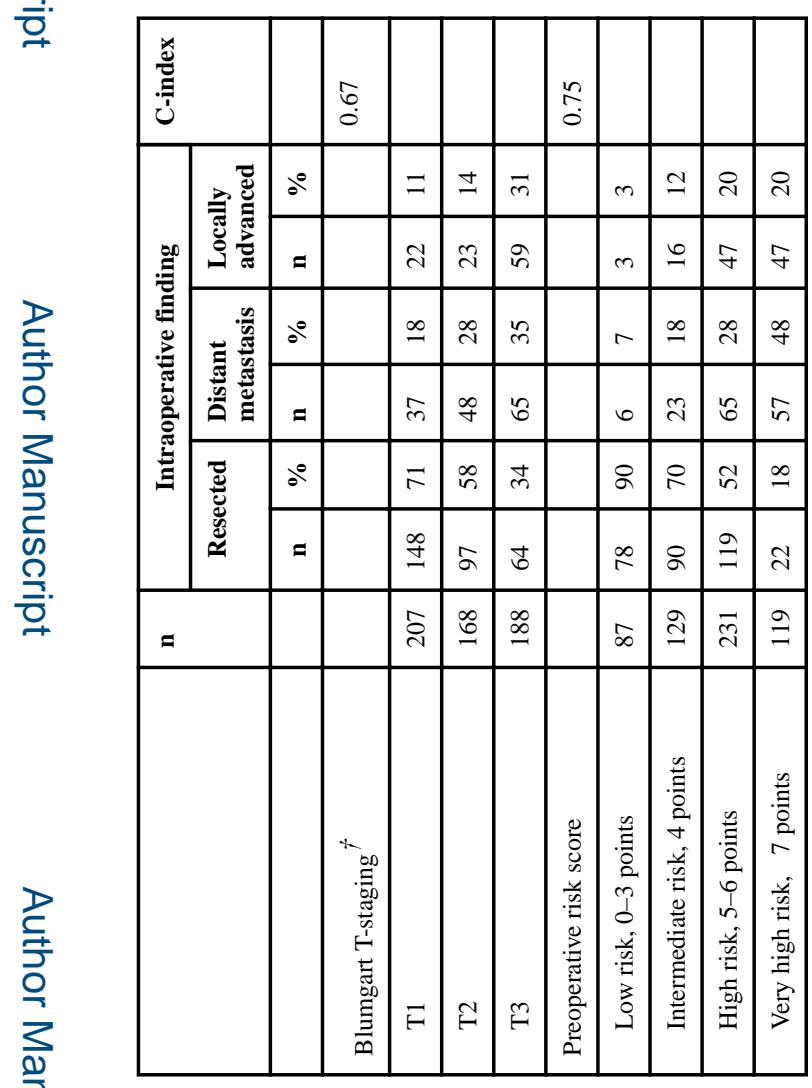

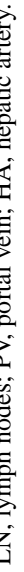


eTable 1.

Full Multivariable Analysis of Preoperative Predictors of Occult Distant Metastatic or Locally Advanced Disease in Patients with Resectable Perihilar Cholangiocarcinoma on Imaging $(n=566)$

\begin{tabular}{|c|c|c|c|c|c|}
\hline Variable & $\mathbf{n}$ & $\%$ & Odds ratio & $95 \% \mathrm{CI}$ & $\begin{array}{c}\text { p } \\
\text { Value }\end{array}$ \\
\hline Age per 10 years & NA & & 1.02 & $1.00-1.03$ & 0.087 \\
\hline Male & 351 & 62.0 & 0.87 & $0.58-1.31$ & 0.507 \\
\hline Bilirubin $>2 \mathrm{mg} / \mathrm{dL}$ & 459 & 81.1 & 1.88 & $1.08-3.27$ & 0.026 \\
\hline \multicolumn{6}{|l|}{ Bismuth classification on imaging ${ }^{*}$} \\
\hline Left or right hepatic duct only & 35 & 6.2 & 0.46 & $0.12-1.71$ & 0.246 \\
\hline B1 & 69 & 12.2 & Reference & - & - \\
\hline $\mathrm{B} 2$ & 55 & 9.7 & 2.26 & $1.21-9.4$ & 0.03 \\
\hline B3A, right & 172 & 30.4 & 2.51 & $1.51-8.32$ & 0.007 \\
\hline B3B, left & 109 & 19.2 & 2.18 & $1.29-7.85$ & 0.02 \\
\hline B4 & 126 & 22.3 & 3.38 & $1.54-7.39$ & 0.002 \\
\hline \multicolumn{6}{|l|}{ PV involvement on imaging ${ }^{\dagger \%}$} \\
\hline None & 268 & 47.3 & Reference & - & - \\
\hline Unilateral & 215 & 38.0 & 1.48 & $0.88-2.51$ & 0.143 \\
\hline Bilateral or main & 62 & 11.0 & 6.17 & $2.72-13.99$ & $<0.001$ \\
\hline \multicolumn{6}{|l|}{ HA involvement on imaging ${ }^{t+t}$} \\
\hline None & 330 & 58.3 & Reference & - & - \\
\hline Unilateral & 169 & 29.9 & 1.68 & $1.06-2.65$ & 0.027 \\
\hline Bilateral or main & 31 & 5.5 & 13.28 & $2.44-72.13$ & 0.003 \\
\hline \multicolumn{6}{|l|}{ Suspicious LN on imaging ${ }^{t}$} \\
\hline None & 290 & 51.2 & Reference & - & - \\
\hline $\mathrm{N} 1$ & 199 & 35.2 & 2.03 & $1.34-3.07$ & 0.001 \\
\hline $\mathrm{N} 2$ & 55 & 9.7 & 3.00 & $1.54-5.86$ & 0.001 \\
\hline Lobar atrophy on imaging ${ }^{t}$ & 189 & 33.4 & 0.65 & $0.39-1.08$ & 0.098 \\
\hline \multicolumn{6}{|l|}{ Tumor size on imaging ${ }^{t}$} \\
\hline$<1 \mathrm{~cm}$ & 61 & 10.8 & Reference & - & - \\
\hline $1-3 \mathrm{~cm}$ & 292 & 51.6 & 1.21 & $0.63-2.34$ & 0.567 \\
\hline$>3 \mathrm{~cm}$ & 181 & 32.0 & 1.21 & $0.59-2.50$ & 0.600 \\
\hline
\end{tabular}

Variables are shown as the number of patients $(\%)$, unless stated otherwise.

* Bismuth classification describes the extent of bile duct involvement.

${ }^{\dagger}$ Vascular involvement (portal vein or hepatic artery) on imaging was defined as occlusion, narrowing, or circumferential tumor contact of at least 180 degrees.

${ }^{5}$ Numbers do not sum to group totals due to missing data. In the analysis, missing values have been handled with multiple imputation.

NA, not applicable; LN, lymph nodes; PV, portal vein; HA, hepatic artery. 